\title{
Modelos para a Construção de Siste- mas Multiagentes: Um Estudo de Caso em Sistemas Tutores Inteligentes
}

\author{
Marlos Tacio Silva \\ UFAL - Av. Lourival Melo Mota, s/n, \\ Tabuleiro do Martins / CEP 57072- \\ 970 - Maceió (AL) \\ GrOW - Grupo de Otimização na \\ Web \\ marlos.tacio@gmail.com
}

\author{
Ig Ibert Bittencourt \\ UFAL - Av. Lourival Melo Mota, s/n, \\ Tabuleiro do Martins / CEP 57072- \\ 970 - Maceió (AL) \\ GrOW - Grupo de Otimização na \\ Web \\ ig.ibert@ic.ufal.br
}

\author{
Evandro de Barros Costa \\ UFAL - Av. Lourival Melo Mota, s/n, \\ Tabuleiro do Martins / CEP 57072- \\ 970 - Maceió (AL) \\ GrOW - Grupo de Otimização na \\ Web \\ ebcosta@gmail.com
}

\begin{abstract}
Resumo Este trabalho apresenta modelos para a construção de sistemas multiagentes. Primeiramente, apresenta-se um modelo para a especificação de sistemas multiagentes baseado na metodologia Gaia. Adiante apresenta-se um modelo para verificação do sistema baseado em redes de Petri de alto nível. Para a avaliação dos modelos é apresentado um estudo de caso em Sistemas Tutores Inteligentes no domínio de Matemática. Este estudo de caso utiliza a visão multidimensional do modelo Mathema para a especificação do domínio e, a partir deste, os modelos de especificação e verificação propostos aqui. Com isso, visa-se ter um conjunto de modelos formais que possam ser mais facilmente reutilizados e automaticamente processados.
\end{abstract}

Palavras-Chave: Sistemas Multiagentes, Representação de Conhecimento, Verificação de Modelos, Sistemas Tutores Inteligentes

\begin{abstract}
This paper presents models for building multi-agent systems. First, we present a model for specifying multi-agent systems based on the Gaia methodology. Below we present a model for system verification based on the high level Petri net. For the evaluation of the models we present a case study in Intelligent Tutoring Systems in the domain of mathematics. In this case study is used a multidimensional view of Mathema model for specifying the domain and, finally, we use the specification and verification models. Thus, the aim is to have a set of formal models that can be more easily reused and automatically processed.
\end{abstract}

Keywords: Multiagent Systems, Knowledge Representation, Model Verification, Intelligent Tutoring Systems 


\section{Introdução}

Ao longo dos anos as crescentes inovações tecnológicas (e.g. ubiquidade, interconexão, inteligência, delegação, entre outros) vêm tornando a construção de software de qualidade uma tarefa cada vez mais árdua [1]. Nesse contexto, o papel da Engenharia de Software é o de conceber técnicas que visam a diminuição dessa complexidade $^{1}$ a fim de viabilizar o ciclo de desenvolvimento desses softwares [2]. Por conseguinte, inúmeros paradigmas (e.g., programação orientada a objetos, padrões de projeto, programação baseada em componentes, arquitetura orientada a serviços etc.) têm sido propostos para prover diferentes formas de abstração objetivando a diminuição da complexidade na construção de software.

Dando continuidade, pesquisadores renomados da academia (e.g., [2], [3], [4], [5], [6]) têm apontado a abordagem orientada a agentes como adequada em face à crescente complexidade dos sistemas, pois esse apresentam características como, autonomia, habilidade social, orientação a objetivos, entre outras. Em suma, a abordagem orientada agentes define aplicações em função de entidades de software autônomas que estão situadas em um ambiente e que visam atingir seus objetivos interagindo entre si por meio de protocolos de alto nível.

Embora a abordagem orientada a agentes tenha como objetivo prover uma forma diferente de analisar, projetar e desenvolver soluções para aplicações complexas, é preciso lidar com uma série de abstrações e técnicas utilizadas no desenvolvimento desses sistemas (e.g., agentes, interações, organizações etc.). Diante disto, inúmeras abordagens têm sido propostas (e.g., [7], [8], [9]) visando facilitar as etapas envolvidas no ciclo de vida do software orientado a agentes.

Todavia, a falta de conformidade entre as diferentes abordagens existentes onera o compartilhamento dos resultados de pesquisa e impõe sérios obstáculos na implantação em larga escala da tecnologia de agentes na indústria. Além disso, a maior parte dessas abordagens não provê mecanismos que facilitem o compartilhamento e a extensão dos artefatos construídos e, nem tão pouco, fornecem uma descrição formal dos mesmos, dificultando o processamento automático das informações especificadas.

Por fim, a natureza concorrente, distribuída e dinâmica dos sistemas multiagentes apresenta um grande desafio no que tange a verificação das interações entre os agentes. Além disso, estudos sugerem que o processo de testes em um sistema possa alocar de 25 a 50 por cento do custo

${ }^{1}$ o termo "complexidade" está sendo utilizado para designar a dificuldade inerente ao desenvolvimento de software e não em sua forma técnica, que abrange complexidade computacional ou algorítmica. total do projeto, de modo que grande parte do tempo de desenvolvimento é gasto localizando falhas.

Com base nos problemas apresentados, este trabalho descreve a construção de dois modelos: modelo de especificação e modelo de verificação. O primeiro tem o papel de prover uma base formal para a especificação de sistemas multiagentes com base em alguma metodologia existente. $\mathrm{O}$ segundo tem a função de prover uma base para a validação do sistema especificado pelo modelo anterior. Para a avaliação dos modelos será apresentado um estudo de caso de um Sistema Tutor Inteligente no domínio de Matemática. Este estudo de caso foi construído com base no modelo Mathema [10], que é um modelo para a construção de sistemas tutores inteligentes.

O conteúdo deste artigo está estruturado em seções, sendo a presente a primeira. Na Seção 2, é apresentado o modelo Mathema, que servirá para o entendimento do estudo de caso. Na Seção 3, são abordados os trabalhos relacionados a esta proposta. Na Seção 4, é mostrada solução proposta por este trabalho. Na Seção 5, é inserido o estudo de caso em Ambientes Interativos de Aprendizagem, mais especificamente no domínio de Matemática. Por fim, na Seção 6, são apresentadas as conclusões do trabalho e as direções futuras que o mesmo pode seguir.

\section{O modelo Mathema}

O modelo conceitual do Mathema [10] é proposto com base na arquitetura de um Sistemas Tutor Inteligente. O modelo define uma sociedade de agentes tutores inteligentes de modo que cada agentes foca um subdomínio específico. Para a modelagem do domínio, que é o foco desse trabalho, o modelo se divide em duas visões: visão externa e visão interna.

A visão externa particiona o domínio de conhecimento e sob uma perspectiva tridimensional:

- Contexto: um domínio de conhecimento pode ser composto por diferentes contextos ou pontos de vistas. Em outras palavras, um domínio de conhecimento pode ter diferentes abordagens de ensino;

- Profundidade: dado um contexto particular, este pode ser dividido de acordo com níveis de aprofundamento do conhecimento;

- Lateralidade: dado um contexto particular e um profundidade particular, conhecimentos complementares podem ser necessários para o completo entendimento. Em outra palavras é um conhecimento que está fora do escopo do domínio. 
Portanto, com esta abordagem multidimensional (vide Figura 1) é possível focar um domínio via uma visão contextual, e essa visão pode definir diversos níveis de aprofundamento . Além disso, essa estrutura define uma terceira dimensão que é o conhecimento que está fora do escopo do domínio de conhecimento, mas que se faz necessário na construção do conhecimento.

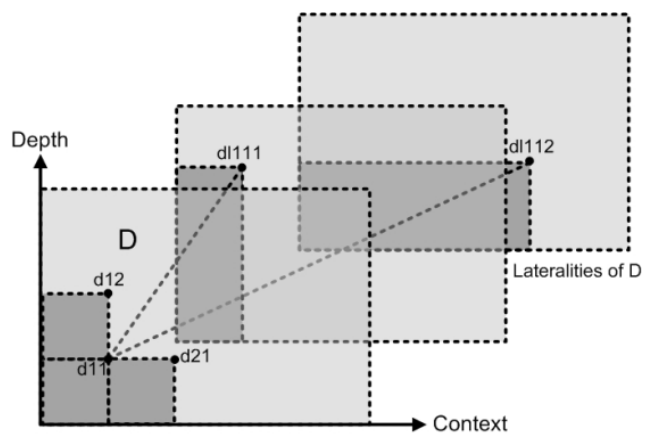

Figura 1:Visão multidimensional de um domínio de conhecimento

Uma vez definida a visão externa, uma visão interna pode ser aplicada a cada subdomínio. Na construção da visão interna, o conhecimento associada a cada subdomínio deve ser organizado em um conjunto de currículos. Cada currículo é progressivamente refinado de acordo com três níveis:

- Nível 1: cada currículo é dividido em um conjunto de unidades pedagógicas. Essas unidades pedagógicas estão relacionadas com base em alguma ordem prédefinida;

- Nível 2: cada unidade pedagógica é refinada em um conjunto de problemas, que podem estar ordenados segundo algum critério;

- Nível 3: cada problema é associado a um conjunto de unidades de suporte, de modo que o aprendiz pode utilizá-las na para ajudar a suprir conhecimentos relacionados.

A estrutura pedagógica do domínio possui três planos fundamentais que são denominados: plano pedagógico, plano de problemas e plano de suporte. Como pode ser observado na Figura 2, o plano superior é composto das unidades pedagógicas que estão relacionados segundo alguma ordem. No plano central estão os problemas de modo que cada um está associado a uma unidade pedagógica, servindo como uma atividade pedagógico básica no processo de ensino/aprendizagem. Por fim, no plano inferior é definida a utilização de conhecimento de suporte para apoiar a resolução dos problemas. Nesse plano podem estar incluídos: conceitos, exemplos, dicas, problemas semelhantes, entre outros.

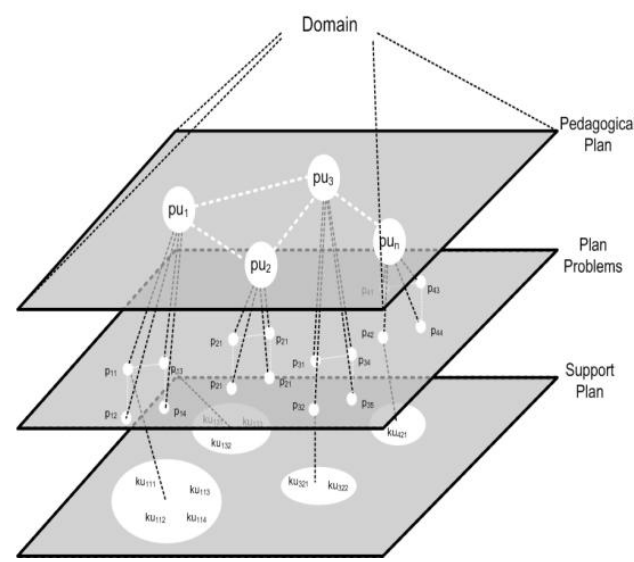

Figura 2: Estrutura pedagógica de um domínio de conhecimento

Exemplo: Domínio da Lógica Clássica

Suponha que o domínio a ser modelado seja o da Lógica Clássica. Definindo este domínio de acordo com o modelo multidimensional de conhecimento apresentado anteriormente, pode-se considerar as seguintes dimensões:

- Contextos

$$
\begin{array}{ll}
\circ & C_{1}=\text { uma visão axiomática; } \\
\circ & C_{2}=\text { uma visão via Dedução Natural } \\
\circ & C_{3}=\text { uma algébrica (Semântica). }
\end{array}
$$

Os três contextos acima representados são equivalentes no que se refere ao conjunto de fórmulas que eles geram, ou seja, o conjunto de teoremas (gerado pelos dois primeiros) e o de tautologias ou fórmulas válidas (gerado pelo sistema algébrico ou semântico), são os mesmos.

Fixando-se, por exemplo, $C_{1}$, poderiam-se ter as seguintes profundidades:

\section{- Profundidades}

$$
\begin{aligned}
& \text { - } \quad P_{11}=\text { Lógica Clássica Proposicional; } \\
& \text { - } \quad P_{12}=\text { Lógica Clássica de Predicados }
\end{aligned}
$$

Em relação ao par $\left\langle C_{1}, P_{11}\right\rangle$, pode-se definir as seguintes lateralidades:

\section{- $\quad$ Lateralidades}

$$
\begin{aligned}
\circ & L_{111}=\text { Teoria dos Conjuntos; } \\
\circ \quad L_{112} & =\text { Princípio da Indução Finita.. }
\end{aligned}
$$

\section{Trabalhos Relacionados}

Esta seção tem o objetivo de apresentar os trabalhos que se relacionam com esta proposta. Durante o processo, foram reunidos propostas que utilizam rede de Petri para a representação e validação de sistemas multiagentes. 
Cabac e Moldt [10] propõem o uso de redes de Petri para a modelagem de interação entre agentes utilizando redes baseadas na linguagem AUML. Para isso, definem um conjunto de redes básicas (e.g. redes condicionais, redes de repetição, rede de paralelismo) que tornam a criação da redes de Petri mais intuitiva.

Bai, Zhang, and Win [11] apresentam uma abordagem para definir protocolos de interações entre agentes baseado em redes de Petri coloridas. Esta proposta visa flexibilizar as interações entre agentes utilizando uma estrutura pré-definida de rede que analisa o tipo de dado recebido para tomar uma determinada ação.

Nowostawski, Purvis, e Cranefield [12] definem uma arquitetura em camadas baseada em redes de Petri coloridas para a modelagem de interações entre agentes. Esta arquitetura modela seus interações com base nos protocolos definidos pela FIPA $^{1}$ no intuito de separar a lógica da rede da estrutura dos protocolos, esta arquitetura prevê três camadas: i) camada de protocolo, define o modelo do protocolo; ii) camada de conversação, define instância de um protocolo de interação; e iii) camada de política, define estratégias e restrições específicas de cada interação.

Yu e Cai [13] propõe uma linguagem para a descrição arquitetural de sistema multiagentes baseada em redes de Petri, para a análise de sistemas multiagentes. Esta proposta contempla tanto o nível do agente quanto o nível de sociedade de agentes.

A maioria dos trabalhos focam no problema da comunicação entre agentes [11], [12], [13] e não abordam o processamento interno do agente. Já em [14] é abordada uma visão tanto a nível de agente quanto a nível de sociedade, contudo não existe qualquer tipo de agrupamento entre agentes com objetivos afins. Além disso nenhuma das abordagens utiliza ontologias para representação do conhecimento, o que onera o processamento, compartilhamento e reutilização de suas informações.

Diferentemente dos outros trabalhos, esta abordagem prevê o uso da rede de Petri tanto para a modelagem a nível macro quanto a nível micro agente. Além disso, esta abordagem utiliza ontologias para a especificação do sistema, o que facilita o reuso, o compartilhamento e processamento automático das informações, e regras para geração das redes de Petri.

\section{Proposta}

Com base nos problemas apresentados na Seção 1, é preciso definir um conjunto de modelos que possa ser facilmente processado por entidades de software. Consequentemente, os modelos propostos devem ser descritos

\footnotetext{
${ }^{1}$ Disponível em www.fipa.org (último acesso em 20 de abril de 2011).
}

em uma linguagem formal que proveja expressividade adequada à representação dos mesmos, mas que ao mesmo tempo garanta a decidibilidade no processamento de suas informações.

Diante dos requisitos, ontologias apresentam uma abordagem vantajosa no que diz respeito à representação de conhecimento. A utilização de ontologias provê a explicitação do conhecimento de modo não ambíguo, podendo ser mais facilmente processados por entidades de software. Além disso, o conhecimento representado pode ser compartilhado, reutilizado e estendido. Por conseguinte, o conceito de ontologias pode ser extrapolado para o contexto da Engenharia de Software e, assim, utilizado para representação de conhecimento contido em processos de construção de software. Desse modo, é possível definir um conjunto de artefatos de software que pode ser mais facilmente processado e reutilizado, apresentando uma alternativa promissora com relação ao compartilhamento de conhecimento entre os stakeholders do sistema.

Com base no exposto, as subseções que se seguem visam apresentar a construção dos modelos que contemplam as fases de especificação e verificação do software orientado a agentes. Tais modelos têm o objetivo de prover uma representação formal de conhecimento que doravante serão utilizados como repositórios de conhecimento na construção de sistemas baseados em agentes. Desse modo, a utilização desses modelos não tem o objetivo de ser uma solução definitiva no processo de construção de agentes e nem tão pouco abranger todas as nuances que esses tipos de sistemas apresentam.

\subsection{Modelo de Especificação}

A metodologia GAIA [7] adota um conjunto de terminologias e abstrações visando a construção de sistemas multiagentes em função de uma sociedade organizada de indivíduos de modo que cada agente apresenta um papel específico e interage com outros agentes de acordo com protocolos predeterminados. A metodologia visa prover uma especificação que seja tecnologicamente neutra e que possa ser implementada utilizando algum framework para construção de agentes (e.g., Jade [14]).

GAIA define o processo de especificação do sistema em três etapas:

- Fase de Análise: essa etapa tem o intuito de coletar e especificar os artefatos que serão utilizados para desenvolver o modelo arquitetural do sistema. Nesta primeira fase busca-se definir um modelo que seja independente de topologia organizacional, postergando algumas decisões para a fase arquitetural, onde será definida a estrutura organizacional do sistema; 
- Fase Arquitetural: nessa fase busca-se definir a estrutura organizacional que melhor se aplica ao sistema modelado e, uma vez escolhida a configuração do sistema, completar os detalhes que não puderam ser feitos na fase de análise;

- Fase de Projeto: nessa etapa visa-se definir os agentes que farão parte do sistema e quais os serviços providos por eles.

Ao término do processo, a metodologia produz um conjunto de artefatos que visam auxiliar os desenvolvedores de software no processo de implementação do sistema.

\subsubsection{Formalização do Modelo}

Com base na metodologia proposta por Zambonelli, Jennings e Wooldridge [7], um sistema multiagentes pode ser formalizado segundo as definições a seguir:

Definição 1. Seja $S$ um sistema multiagentes sobre o qual pretende-se construir uma especificação (de acordo com a metodologia descrita anteriormente). Um enfoque para $S$ pode ser formalmente definido assim:

i) A $S$, associa-se um conjunto de organizações distintas $\left\{o_{1}, o_{2}, \cdots, o_{n}\right\}$, onde cada $o_{i}$, com $1 \leq i \leq n$, representa uma organização particular. Isto é:

$$
S \rightarrow\left\{o_{1}, o_{2}, \cdots, o_{n}\right\}
$$

ii) A $S$, associa-se um conjunto de agentes distintos $\left\{a g_{1}, a g_{2}, \cdots, a g_{m}\right\}$, onde cada $a g_{j}$, com $1 \leq j \leq$ $m$, representa um agente particular. Isto é:

$$
S \rightarrow\left\{a g_{1}, a g_{2}, \cdots, a g_{m}\right\}
$$

iii) A $S$, associa-se um conjunto de interações distintas $I=\left\{i_{1}, i_{2}, \cdots, i_{t}\right\}$, onde cada $i_{k}$, com $1 \leq k \leq t$, representa uma interação particular. Isto é:

$$
S \rightarrow\left\{i_{1}, i_{2}, \cdots, i_{t}\right\}
$$

iv) A $S$, associa-se um conjunto de organizações estruturais distintas $E=\left\{e_{1}, e_{2}, \cdots, e_{u}\right\}$, onde cada $e_{l}$, com $1 \leq l \leq u$, representa uma interação particular. Assim:

$$
S \rightarrow\left\{e_{1}, e_{2}, \cdots, e_{u}\right\}
$$

Desse modo, temos que um sistema é formado por um conjunto de organizações, que dividem o sistema em módulos. Adicionalmente, um sistema é composto por um conjunto de agentes e interações, que definem as entidades do sistema e quais as interações entre elas. Por fim, tem-se um conjunto de organizações estruturais, que definem a estrutura hierárquica entre as entidades, em outras palavras, a arquitetura do sistema em si.
Definição 2. Seja $o_{i}$ uma organização fixada qualquer, temos que esta pode ser formalmente definida assim:

i) $\mathrm{A} \quad o_{i}$, atribui-se um conjunto de papéis $\left\{p_{i 1}, p_{i 2}, \cdots, p_{i a}\right\}$, onde $p_{i b}$, com $1 \leq a \leq b$, representa um papel particular. Isto é:

$$
o_{i} \rightarrow\left\{p_{i 1}, p_{i 2}, \cdots, p_{i a}\right\}
$$

ii) A cada par $\left\langle o_{i}, p_{i b}\right\rangle$, associa-se um conjunto de permissões $\left\{p e_{i b 1}, p e_{i b 2}, \cdots, p e_{i b c}\right\}$, onde cada $p e_{i b d}$, com $d=1$, representa a d-ésima permissão associada ao par. Isto é:

$$
\left\langle o_{i}, p_{i b}\right\rangle \rightarrow\left\{p e_{i b 1}, p e_{i b 2}, \cdots, p e_{i b c}\right\}
$$

iii) A cada par $\left\langle o_{i}, p_{i b}\right\rangle$, associa-se um conjunto de responsabilidades $\left\{r_{i b 1}, r_{i b 2}, \cdots, r_{i b g}\right\}$, onde cada $r_{i b h}$, com $h=1$, representa a h-ésima responsabilidade associada ao par. Isto é:

$$
\left\langle o_{i}, p_{i b}\right\rangle \rightarrow\left\{r_{i b 1}, r_{i b 2}, \cdots, r_{i b g}\right\}
$$

iv) A cada trípla $\left\langle o_{i}, p_{i b}, p e_{i b d}\right\rangle$, associa-se um conjunto de recursos $\left\{r c_{i b d 1}, r c_{i b d 2}, \cdots, r c_{i b d e}\right\}$, onde $r c_{i b d f}$, $\operatorname{com} f=1$, representa o f-ésimo recurso associado à tripla. Isto é:

$$
\left\langle o_{i}, p_{i b}, p e_{i b d}\right\rangle \rightarrow\left\{r c_{i b d 1}, r c_{i b d 2}, \cdots, r c_{i b d e}\right\}
$$

v) A cada tripla $\left\langle o_{i}, p_{i b}, r_{i b h}\right\rangle$, associa-se um conjunto de ações $\left\{a c_{i b h 1}, a c_{i b h 2}, \cdots, a c_{i b h v}\right\}$, onde $a c_{i b h o}$, com $o=1$, representa a o-ésima ação associada à trípla. Isto é:

$$
\left\langle o_{i}, p_{i b}, r_{i b h}\right\rangle \rightarrow\left\{a c_{i b h 1}, a c_{i b h 2}, \cdots, a c_{i b h v}\right\}
$$

vi) Seja pe o conjunto de todas as permissões, tem-se que os elementos desse conjunto pertencem ao subconjunto \{leitura, escrita, consumo\}. Isto é:

$$
\text { pe } \supseteq\{\text { leitura, escrita, consumo }\}
$$

vii) Seja $r$ o conjunto de todas as responsabilidades, temse que os elementos desse conjunto pertencem ao subconjunto $\{$ vital, segurança $\}$. Isto é:

$$
r \supseteq\{\text { vital, segurança\} }
$$

viii) Seja $a c$ o conjunto de todas as ações, tem-se que os elementos desse conjunto pertencem ao subconjunto \{atividade, protoclo $\}$. Isto é:

$$
\text { ac } \supseteq\{\text { atividade, protocolo }\}
$$

Em suma, uma organização é composta por um conjunto de papéis. Aos papéis estão associadas um conjunto de permissões e de responsabilidades. As permissões definem restrições de acesso (e.g., permissões de leitura, permissões de escrita e permissões de consumo) aos recursos disponíveis. Cada papel conta com um conjunto 
de responsabilidades que definem quais as ações que determinado papel deve desempenhar. As ações podem ser de dois tipos: atividades, que representam ações que não necessitam da interação de terceiros; e protocolos, que representam ações que necessitam da ação de outros papéis.

Definição 3. Seja $i_{j}$ uma interação qualquer, temos que essa pode ser formalmente definida assim:

i) A $i_{j}$, associa-se um par de papéis distintos $\left\langle p_{r s}, p_{x z}\right\rangle$, onde $p_{r s}$, com $s=1$, representa o s-ésimo papel associado a r-ésima organização, ou o papel remetente, e onde $p_{x z}$, com $z=1$, representa z-ésimo papel associado a z-ésimo organização, ou o papel destinatário. Isto é:

$$
i_{j} \rightarrow\left\langle p_{r s}, p_{x z}\right\rangle \text {, onde }\left\{P_{r s}\right\} \cap\left\{P_{x z}\right\}=\varnothing
$$

ii) A tripla $\left\langle i_{j}, p_{r s}, p_{x z}\right\rangle$, associa-se dois conjunto de recursos distintos $\left\{r e_{r s 1}, r e_{r s 2}, \cdots, r e_{r s \alpha}\right\}$, onde $r e_{r s \beta}, \operatorname{com} \beta=1$, representa o $\beta$-ésimo recurso associado ao s-ésimo papel de uma organização, ou se$\mathrm{ja}$, os recursos de entrada, e $\left\{r e_{x z 1}, r e_{x z 2}, \cdots, r e_{x z \gamma}\right\}$, onde $r e_{r s \delta}$, com $\delta=1$, representa o $\delta$-ésimo recurso associado ao z-ésimo papel de uma organização, ou seja, os recursos de saída. Isto é:

$$
\begin{aligned}
\left\langle i_{j}, p_{r s}, p_{x z}\right\rangle \rightarrow\left\{r e_{r s 1}, r e_{r s 2}, \cdots, r e_{r s \alpha}\right\} & \left.r e_{x z \gamma}\right\}
\end{aligned}
$$

Cada interação é composto por dois papéis, um responsável por iniciar uma conversa e outro por respondela. Para cada interação, podem ser utilizados recursos do ambiente, tanto para o envio de recursos (conjunto de recursos de entrada) quanto para o recebimento de recursos (conjunto de recursos de saída).

Definição 4. Seja $e_{l}$ uma estrutura organizacional qualquer, temos que essa pode ser formalmente definida assim:

i) $\mathrm{A} e_{l}$ associa-se um par de papéis distintos $\left\langle p_{\zeta \varepsilon}, p_{\eta \theta}\right\rangle$, onde $p_{\zeta \varepsilon}$, $\operatorname{com} \varepsilon=1$, representa o $\varepsilon$-ésimo papel associado a $\zeta$-ésima organização, ou o papel primário, e onde $p_{\eta \theta}, \operatorname{com} \theta=1$, representa $\theta$-ésimo papel associado a $\eta$-ésimo organização, ou o papel secundário. Isto é:

$$
e_{l} \rightarrow\left\langle p_{\zeta \varepsilon}, p_{\eta \theta}\right\rangle, \text { onde }\left\{p_{\zeta \varepsilon}\right\} \cap\left\{p_{\eta \theta}\right\}=\emptyset
$$

ii) Dado um conjunto e de estruturas organizacionais, tem-se todos os elementos desse conjunto pertencem ao subconjunto \{igualdade, controle \}. Isto é:

$$
e \supseteq\{\text { igualdade, controle }\}
$$

Desse modo, tem-se que dois papéis estão relacionados por meio de uma estrutura organizacional, isto é, um papel pode ter um relacionamento de igualdade para com outro papel, ou então, ter o controle sobre as ações de outro papel.

Definição 5. Seja $a g_{k}$ um agente, temos que esse pode ser formalmente definido assim:

i) A $a g_{k}$, associa-se um conjunto de papéis $\left\{p_{i 1}, p_{i 2}, \cdots, p_{i a}\right\}$, onde cada $p_{i b}$, com $1 \leq a \leq b$, representa um papel particular. Isto é:

$$
a g \rightarrow\left\{p_{1}, p_{2}, \cdots, p_{n}\right\}
$$

ii) A $a g_{k}$, associa-se um conjunto de serviços $\left\{s e_{k 1}, s e_{k 2}, \cdots, s e_{k \omega}\right\}$, onde cada $s e_{k \phi}, \operatorname{com} \phi=1$, representa o $\phi$-ésimo serviço associado a determinado agente. Isto é:

$$
a g_{k} \rightarrow\left\{s e_{k 1}, s e_{k 2}, \cdots, s e_{k \omega}\right\}
$$

iii) Ao par $\left\langle a g_{k}, s e_{k \phi}\right\rangle$, associam-se dois conjuntos de recursos distintos $\left\{R e_{1}, R e_{2}, \cdots, R e_{n}\right\}$, onde cada $R e_{i}$, com $1 \leq i \leq n$, representa os recursos de entrada, e $\left\{R e_{1}, R e_{2}, \cdots, R e_{m}\right\}$, onde cada $R e_{j}$, com $1 \leq j \leq m$, representa os recursos de saída. Isto é:

$$
\begin{gathered}
\left\langle a g_{k}, s e_{k \phi}\right\rangle \rightarrow\left\{\operatorname{Re}_{1}, \operatorname{Re}_{2}, \cdots, \operatorname{Re}_{n}\right\} \\
\cup\left\{\operatorname{Re}_{1}, \operatorname{Re}_{2}, \cdots, \operatorname{Re}_{m}\right\}
\end{gathered}
$$

iv) Ao par $\left\langle\boldsymbol{a g}_{\boldsymbol{k}}, \boldsymbol{s} \boldsymbol{e}_{\boldsymbol{k} \boldsymbol{\phi}}\right\rangle$, associam-se dois conjunto de expressões distintos $\left\{\boldsymbol{E} \boldsymbol{x}_{\mathbf{1}}, \boldsymbol{E} \boldsymbol{x}_{\mathbf{2}}, \cdots, \boldsymbol{E} \boldsymbol{x}_{\boldsymbol{t}}\right\}$, onde cada $\boldsymbol{E} \boldsymbol{x}_{\boldsymbol{k}}$, com $1 \leq \boldsymbol{k} \leq \boldsymbol{t}$, representa as pré-condições, e $\left\{\boldsymbol{E} \boldsymbol{x}_{1}, \boldsymbol{E} \boldsymbol{x}_{2}, \cdots, \boldsymbol{E} \boldsymbol{x}_{\boldsymbol{u}}\right\}$, onde cada $\boldsymbol{E} \boldsymbol{x}_{\boldsymbol{l}}$, com $\mathbf{1} \leq \boldsymbol{l} \leq$ $\boldsymbol{l}$, representa as pós-condições. Isto é:

$$
\begin{gathered}
\left\langle\boldsymbol{a g}_{k}, \boldsymbol{s e}_{\boldsymbol{k} \phi}\right\rangle \rightarrow\left\{E x_{1}, E x_{2}, \cdots, E x_{t}\right\} \\
\cup\left\{E x_{1}, E x_{2}, \cdots, E x_{u}\right\}
\end{gathered}
$$

Assim, um conjunto de agentes desempenha um conjunto de papéis e provê uma série de serviços. Os serviços são compostos por recursos de entrada e saída e, além disso, pode ser composto por pré e pós condições.

\subsection{Modelo de Verificação}

Redes de Petri são uma ferramenta de modelagem tanto gráfica quanto matemática que pode ser aplicada em muitos sistemas. São uma ferramenta promissora para descrever e estudar informações do processamento de sistemas caracterizados por serem concorrentes, assíncronos, distribuídos, paralelos e não-determinísticos [16]. Além disso, são interessantes pois podem ser utilizadas tanto por práticos quanto por teóricos e pode ser visto como um poderoso meio de comunicação entre estes. Assim, tanto práticos podem aprender como fazer seus modelos mais teóricos, como os teóricos podem aprender como fazer seus modelos mais realistas.

O padrão de redes de Petri de alto nível [17], visa definir um conjunto de terminologias para descrição tanto sintática quanto semântica das rede de Petri de Alto Ní- 
vel. Com isso, visa prover um modelo de referência com o objetivo facilitar a interoperabilidade entre diferentes ferramentas de desenvolvimento. O modelo hierárquico de rede de Petri é baseado no trabalho de Kurt Jensen [18] e tem o objetivo de definir hierarquias em uma rede de Petri. Essas hierarquias funcionam como módulos e dividem o sistema para facilitar a visualização da mesma.

\subsubsection{Formalização do Modelo de Alto Nível}

Com base na padrão proposto em [17], uma rede de Petri pode ser formalizado segundo as definições a seguir:

Definição 6. Seja $R$ uma rede de Petri de alto nível sobre o qual pretende-se construir uma especificação (de acordo com o padrão descrito anteriormente). Um enfoque para $R$ pode ser formalmente definido as$\operatorname{sim}$ :

i) A $R$, associa-se um conjunto de nós distintos $\left\{n_{1}, n_{2}, \cdots, n_{n}\right\}$, onde cada $n_{i}$, com $1 \leq i \leq n$, representa um nó particular. Isto é:

$$
R \rightarrow\left\{n_{1}, n_{2}, \cdots, n_{n}\right\}
$$

ii) A $R$, associa-se um conjunto de arcos distintos $\left\{a_{1}, a_{2}, \cdots, a_{m}\right\}$, onde cada $a_{i}$, com $1 \leq j \leq m$, representa um arco particular. Isto é:

$$
R \rightarrow\left\{a_{1}, a_{2}, \cdots, a_{m}\right\}
$$

iii) A $R$, associa-se um conjunto de variáveis distintas $\left\{v_{1}, v_{2}, \cdots, v_{l}\right\}$, onde cada $v$, com $1 \leq l \leq k$, representa uma variável particular. Isto é:

$$
R \rightarrow\left\{v_{1}, v_{2}, \cdots, v_{l}\right\}
$$

Assim, uma rede de Petri de alto nível pode ser visto como um grafo composto por nós, arcos e variáveis. Os nós e arcos serão utilizados para definir a estrutura em grafo da rede e as variáveis poderão ser utilizadas no processamento de nós e/ou arcos.

Definição 7. Seja $n_{i}$ um nó particular qualquer, temos que esse pode ser formalmente definido assim:

i) Seja $n$ o conjunto formado pela união de todos os nós, temos que esse conjunto é composto pela união do subconjunto de lugares $\left\{l_{i 1}, l_{i 2}, \cdots, l_{i a}\right\}$, onde cada $l_{i b}$, com $1 \leq a \leq b$, representa um lugar particular e por um subconjunto de transições $t=$ $\left\{t_{i 1}, t_{i 2}, \cdots, t_{i c}\right\}$, onde cada $t_{d}$, com $1 \leq c \leq d$, representa uma transição particular. Isto é:

$$
n \subseteq\left\{l_{i 1}, l_{i 2}, \cdots, l_{i b}\right\} \cup\left\{t_{i 1}, t_{i 2}, \cdots, t_{i d}\right\} \text {, sendo } l \cap t=\varnothing
$$

ii) A cada $l_{i b}$, associa-se um tipo $t p_{e}$, onde $1 \leq e \leq f$, representa um tipo particular. Isto é:

$$
l_{i b} \rightarrow t p_{e}
$$

i) A cada $t_{i d}$, associa-se uma expressão $e_{g}$, onde $1 \leq g \leq h$, representa uma expressão particular. Isto é:

$$
t_{i d} \rightarrow e_{g}
$$

Desse modo, temos que um nó é um conceito abstrato formado pelo conjunto dos lugares de das transições. Para cada lugar deve ser definido um tipo e para cada transição é definida uma expressão que define as regras de habilitação do disparo.

Definição 8. Seja $a_{j}$ um arco fixado qualquer, temos que esse pode ser formalmente definido assim:

i) A cada $a_{j}$, associa-se um par $\left\langle n_{i o}, n_{i p}\right\rangle$, onde $n_{o}$, com $1 \leq o \leq q$, representa o nó de origem e onde $n_{p}$, com $1 \leq p \leq r$, representa o nó de destino, de modo que um arco deve, obrigatoriamente, estar associado a um lugar e a uma transição. Isto é:

$a_{j} \rightarrow\left\langle n_{i o}, n_{i p}\right\rangle$, onde $n_{o} \in l, n_{p} \in$ t ou $n_{o} \in t, n_{p} \in l$

ii) A cada $a_{j}$, associa-se uma expressão $e_{u}$, onde $1 \leq s \leq u$, representa uma expressão particular. Isto é:

$$
a_{j} \rightarrow e_{u}
$$

Os arcos podem ser vistos como arestas que interligam os nós de um grafo. Nesse caso, temos que um arco deve interligar nós de tipos diferentes, isto é, associar um lugar a uma transição, ou vice-versa. Além disso, cada arco contêm uma expressão que será avaliada na execução da rede.

\subsubsection{Formalização do Modelo Hierárquico}

Com base na trabalho apresentado em [18], uma rede de Petri hierárquica pode ser formalizado segundo as definições a seguir:

Definição 9. Seja $R H$ uma rede de Petri hierárquica sobre o qual pretende-se construir uma especificação. Um enfoque para RH pode ser formalmente definido assim:

i) Tem-se que $R H$, é um subconjunto de $\mathrm{R}$ :

$$
R H \subseteq R
$$

ii) A $R H$, associa-se um conjunto de páginas distintas $\left\{p g_{1}, p g_{2}, \cdots, p g_{n}\right\}$, onde cada $p g_{i}$, com $1 \leq i \leq n$, representa uma página particular. Isto é:

$$
R H \rightarrow\left\{p g_{1}, p g_{2}, \cdots, p g_{n}\right\}
$$

iii) A $R$, associa-se um conjunto de substituições distintas $\left\{s_{1}, s_{2}, \cdots, s_{m}\right\}$, onde cada $s_{j}$, com $1 \leq j \leq m$, representa uma substituição particular. Isto é:

$$
R H \rightarrow\left\{s_{1}, s_{2}, \cdots, s_{m}\right\}
$$


iv) A $R$, associa-se um conjunto de fusões distintas $\left\{f_{1}, f_{2}, \cdots, f_{l}\right\}$, onde cada $f_{k}$, com $1 \leq l \leq k$, representa uma fusão particular. Isto é:

$$
R H \rightarrow\left\{f_{1}, f_{2}, \cdots, f_{l}\right\}
$$

Desse modo, temos que uma rede de Petri hierárquica é um tipo especial de rede de Petri composta por páginas, substituições e fusões. As páginas são módulos que dividem a rede em hierarquias. As substituições são utilizadas para inserir módulos ou páginas em nós específicos da rede. Por fim, as fusões, tem o objetivo de interligar módulos ou páginas.

Definição 10. Seja $p g_{i}$ uma página fixada qualquer. temos que essa pode ser formalmente definida assim:

i) Ao par $\left\langle r h_{a}, p g_{a i}\right\rangle$, onde $r h_{a}$ é uma rede de Petri hierárquica qualquer, associa-se uma rede de Petri $r_{b}$, diferente de $r h_{a}$. Isto é:

$$
\left\langle r h_{a}, p g_{i}\right\rangle \rightarrow r_{b}, \text { sendo } a \neq b
$$

Assim, temos que a uma página está relacionada uma rede de Petri diferente da rede que possui a página. Desse modo, uma página é vista como um módulo para outra rede de Petri. Assim, tem-se o conceito de hierarquia de redes de Petri.

Definição 11. Seja $s_{j}$ uma substituição fixada qualquer. temos que essa pode ser formalmente definida assim:

i) A $s_{j}$, associa-se um nó $n_{d}$, onde $n_{o}$, com $1 \leq c \leq$ d. Isto é:

$$
s_{j} \rightarrow n_{d}
$$

ii) A $s_{j}$, associa-se uma página $p g_{f}$, onde $p g_{f}$, com $1 \leq e \leq f$. Isto é:

$$
s_{j} \rightarrow p g_{f}
$$

iii) A $s_{j}$, associa-se um conjunto de fusões $\left\{f_{1}, f_{2}, \cdots, f_{g}\right\}$, onde cada $f_{h}$, com $1 \leq g \leq h$, representa uma fusão particular. Isto é :

$$
s_{j} \rightarrow\left\{f_{1}, f_{2}, \cdots, f_{g}\right\}
$$

O conceito de substituição é utilizado para inserir páginas em nós específicos da rede. Assim, é possível substituir um nó qualquer, como uma transição, por exemplo, por uma sub-rede de Petri.

Definição 12. Seja $f_{k}$ uma fusão fixada qualquer. temos que essa pode ser formalmente definida assim:

i) Dado uma fusão $f_{k}$ fixada qualquer, associasse um par de nós $\left\langle n_{a p}, n_{b q}\right\rangle$, com $n_{p}$ referente ao par $\left\langle r h_{a}, n_{p}\right\rangle$ e com $n_{q}$ referente à quadrupla $\left\langle r h_{a}, p g_{a i}, r_{b}, n_{b q}\right\rangle$. Isto é:

$$
f_{k} \rightarrow\left\langle n_{a p}, n_{b q}\right\rangle
$$

Por fim, o conceito de fusão é utilizado para conectar redes em hierarquias diferentes. Desse modo, uma informação processada em uma rede de nível hierárquico mais alto pode ser repassada para redes de níveis mais baixo.

\section{Estudo de Caso}

Visando ilustrar a utilização dos modelos apresentados, segue um exemplo da especificação de um ambiente de aprendizagem matemático. Mais especificamente, focalizaremos um domínio particular da álgebra: o estudo das equações de grau 2.

\subsection{Descrição do domínio}

O domínio se insere no campo da álgebra. Definindo este domínio de acordo com o modelo de domínio baseado nas dimensões de conhecimento propostas no Mathema, tem-se a seguinte configuração:

- Domínio

○ $\quad D=$ estudo da equação de grau 2.

\section{- Contextos}

○ $\quad \boldsymbol{C}_{\mathbf{1}}=$ através de métodos algébricos de resolução canônicos (fórmula de Bhaskara e método da redução).

○ $\quad \boldsymbol{C}_{2}=$ através de métodos de resolução do cálculo numérico.

Focalizando contexto $\boldsymbol{C}_{\mathbf{1}}$, foram definidas as profundidades:

- Profundidade

○ $\quad P_{11}=$ no domínio dos reais.

○ $P_{12}=$ no domínio dos complexos.

Baseado no contexto fixado e nas suas profundidades associadas, podem-se definir os seguintes subdomínios para $\boldsymbol{D}$ :

\section{- Subdomínios}

○ $\boldsymbol{d}_{\mathbf{1 1}}=\left\langle\boldsymbol{C}_{\mathbf{1}}, \boldsymbol{P}_{\mathbf{1 1}}\right\rangle \therefore \boldsymbol{d}_{\mathbf{1 1}}$ é um estudo da equação de segundo grau no domínio dos reais.

○ $d_{12}=\left\langle C_{1}, P_{12}\right\rangle \therefore d_{11}$ é um estudo da equação de segundo grau no domínio dos complexos.

Por agora, somente o $\boldsymbol{d}_{\mathbf{1 1}}$ está sendo focalizado. As unidades pedagógicas a serem trabalhadas em $\boldsymbol{d}_{\mathbf{1 1}}$ são:

- Definição: equação algébrica de grau 2 (equação do segundo grau) é toda sentença do tipo $\boldsymbol{P}(\boldsymbol{x})=$ $\boldsymbol{Q}(\boldsymbol{x})$, onde $\boldsymbol{P}$ e $\boldsymbol{Q}$ são funções polinomiais de grau 
menor ou igual a 2 , mas pelo menos uma delas deve ter grau igual a dois.

- Redução: toda equação do segundo grau é redutível à forma $a x^{2}+b x+c=0, \operatorname{com} a \neq 0$.

- Classificação: equação completa e incompleta.

- Resolução: resolver uma equação quadrática é obter o seu conjunto verdade que é o conjunto de todas as suas raízes. Dizemos que um número real $\boldsymbol{\alpha}$ é raiz de $\boldsymbol{F}(\boldsymbol{x})=\mathbf{0}$, se e somente se $\boldsymbol{F}(\boldsymbol{\alpha})=\mathbf{0}$.

- Teorema da decomposição: toda equação do segundo grau pode ser decomposta num produto de dois fatores do primeiro do tipo $\boldsymbol{x}-\boldsymbol{r} \cdot \boldsymbol{F}(\boldsymbol{x})=$ $a\left(x-x^{\prime}\right)\left(x-x^{\prime \prime}\right)$.

- Obtenção das raízes:

○ método 1: fórmula de Bhaskara;

○ método 2: Decomposição.

- Relações de Girard: $x^{\prime}+x^{\prime \prime}=-(b / a)$ e $x^{\prime} \cdot x^{\prime \prime}=(c / a)$.

Feito isso, volta-se a considerar a seguir a dimensão de lateralidade, a partir da qual pode-se obter novos domínios para serem tratados relativamente ao domínio das equações

Assim, continuando a ilustração, considere fixado o subdomínio $\boldsymbol{d}_{\mathbf{1 1}}$, serão definidos a seguir as lateralidades correspondentes:

\section{- $\quad$ Lateralidades}

○ $d_{111}=L_{111}$ : expressões algébricas - valor numérico, polinômios, redução de termos semelhantes, grau.

○ $\quad d_{112}=L_{112}$ : operações com polinômios - adição e subtração, multiplicação, divisão por um monômio, potência de polinômios.

○ $d_{113}=L_{113}$ : produtos notáveis / fatoração quadrado de um binômio, produto da forma $(\boldsymbol{a}+\boldsymbol{b})(\boldsymbol{a}-\boldsymbol{b})$, fatoração de polinômios (trinômio do quadrado perfeito, e diferença de dois quadrados), fator comum (agrupamento), fatoração por agrupa- mento, fatoração completa.

○ $d_{\mathbf{1 1 4}}=\boldsymbol{L}_{\mathbf{1 1 4}}$ : frações algébricas - simplificações, redução ao mesmo denominador, adição e subtração de frações, multiplicação, divisão e potenciação.

○ $d_{115}=L_{115}$ : equação do primeiro grau - fracionária, definição, resolução.

\subsection{Definição do Sistema Multiagentes}

Com base na modelagem de domínio, visa-se especificar o sistemas multiagentes via o modelo de agentes proposto. Assim, dado o domínio construído, tem-se que o sistema multiagentes tem a seguinte configuração:

\section{- Sistema Multiagentes}

○ $\quad S=$ estudo da equação de grau 2 .

Para a construção das organizações utilizamos uma correspondência com os contextos identificados na modelagem de domínio:

\section{- Organizações}

○ $\boldsymbol{o}_{\mathbf{1}}=$ métodos algébricos.

○ $\boldsymbol{o}_{2}=$ métodos numéricos.

Focalizando a organização $\boldsymbol{o}_{1}$, podemos identificar os seguintes papéis:

- Papéis

○ $\quad \boldsymbol{p}_{\mathbf{1 1}}=$ tutoramento de equações do segundo grau no domínio dos reais.

○ $\quad \boldsymbol{p}_{\mathbf{1 2}}=$ tutoramento de equações do segundo grau no domínio dos complexos.

Além disso, dada a estrutura de lateralidades definidas anteriormente, podemos identificar os seguintes papéis ao focalizarmos as referentes a organização $\boldsymbol{o}_{\mathbf{1}}$ :

- Papéis derivadas

○ $\quad \boldsymbol{p}_{\mathbf{1 3}}=$ expressões algébricas.

○ $\quad \boldsymbol{p}_{\mathbf{1 4}}=$ operações com polinômios.

○ $\quad \boldsymbol{p}_{\mathbf{1 5}}=$ produtos notáveis.

○ $\boldsymbol{p}_{16}=$ frações algébricas.

○ $\quad \boldsymbol{p}_{\mathbf{1 7}}=$ equação primeiro grau.

Fixando o papel $\boldsymbol{p}_{\mathbf{1 1}}$, podem-se especificar as seguintes responsabilidades desse papel:

\section{- Reponsabilidades}

○ $\quad \boldsymbol{r}_{\mathbf{1 1 1}}=$ tutoramento sobre definição.

○ $\quad \boldsymbol{r}_{\mathbf{1 1 2}}=$ tutoramento sobre redução.

○ $\quad \boldsymbol{r}_{\mathbf{1 1 3}}=$ tutoramento sobre decomposição.

○ $\quad \boldsymbol{r}_{\mathbf{1 1 4}}=$ tutoramento sobre obtenção de raízes.

○ $\quad \boldsymbol{r}_{\mathbf{1 1 5}}=$ tutoramento sobre relações de Giradi.

Fixando o papel $\boldsymbol{p}_{\mathbf{1 1}}$, podem-se especificar os seguintes relacionamentos organizacionais entre papéis:

\section{- Estrutura organizacional}




$$
\begin{array}{ll}
\circ & \boldsymbol{e}_{1}=\left\langle\boldsymbol{p}_{11}, \boldsymbol{p}_{13}\right\rangle, \text { com } \boldsymbol{e}_{1} \in\{\text { igualdade }\} \\
\circ & \boldsymbol{e}_{2}=\left\langle\boldsymbol{p}_{11}, \boldsymbol{p}_{14}\right\rangle, \text { com } \boldsymbol{e}_{2} \in\{\text { igualdade }\} \\
\circ & \boldsymbol{e}_{3}=\left\langle\boldsymbol{p}_{11}, \boldsymbol{p}_{15}\right\rangle, \text { com } \boldsymbol{e}_{3} \in\{\text { igualdade }\} \\
\circ & \boldsymbol{e}_{4}=\left\langle\boldsymbol{p}_{11}, \boldsymbol{p}_{16}\right\rangle, \text { com } \boldsymbol{e}_{4} \in\{\text { igualdade }\} \\
\circ & \boldsymbol{e}_{5}=\left\langle\boldsymbol{p}_{11}, \boldsymbol{p}_{17}\right\rangle, \text { com } \boldsymbol{e}_{5} \in\{\text { igualdade }\}
\end{array}
$$

Dados esse relacionamentos, é possível definir um conjunto de agentes que farão parte do sistema:

\section{- $\quad$ ggentes}

$$
\begin{aligned}
\circ & \boldsymbol{a g}_{\mathbf{1}}=\text { equações de grau } 2 \text { no domínio dos reais } \\
\circ & \boldsymbol{a g}_{\mathbf{2}}=\text { expressões algébricas } \\
\circ & \boldsymbol{a g}_{\mathbf{3}}=\text { operações com polinômios } \\
\circ & \boldsymbol{a g}_{\mathbf{4}}=\text { produtos notáveis } \\
\circ & \boldsymbol{a g}_{\mathbf{5}}=\text { frações algébricas } \\
\circ & \boldsymbol{a g}_{\mathbf{6}}=\text { equações primeiro grau }
\end{aligned}
$$

\subsection{Definição da rede de Petri}

Dada a especificação do sistema, é possível construir uma rede de Petri de acordo com o modelo de verificação. De modo sucinto deve-se construir uma rede hierárquica indo desde o nível hierárquico mais alto, que é o nível de organizações, até o nível mais baixo, que é o nível de serviços. Assim, para a construção da rede de Petri são utilizadas as etapas a seguir:

- Dada uma organização identificada no modelo de especificação, definir uma página correspondente, criando o que será denominado de primeiro nível da rede;

- Para cada página de organização, definir uma subpágina para cada agente pertencente à organização, criando o que será denominado de segundo nível da rede;

- Para cada página de agentes, definir uma subpágina para cada papel pertencente ao agente, criando o que será denominado de terceiro nível da rede;

- Para cada página de papel, definir uma subpágina que represente uma entidade padrão, aqui denominada de atuador, criando o que será denominado de quarto nível da rede;

- Para cada atuador, definir uma transição que represente os serviços de cada agente que execute o papel do atuador, criando o que será denominado de quinto e último nível da rede;

- Definir uma pagina especial para cada nível da rede, que encapsulará a lógica das interações para entre as páginas. Tais páginas funcionarão como escalonado- res e conterão as transições e papéis que definem a lógica de interação entre entidades diferentes.

\section{Conclusões}

O objetivo deste trabalho foi o de propor um conjunto de modelos para a construção de sistemas multiagentes via uma abordagem baseada na combinação da metodologia GAIA e de redes de Petri. Afim de clarificar a aplicação dos modelos, esse trabalho apresentou um estudo de caso em Sistemas Tutores Inteligentes no domínio de Matemática.

O trabalho ainda encontra-se em estágio inicial e, como tal, ainda necessita de alguns melhoramentos. Todavia, os modelos propostos são um direcionamento interessante na construção e verificação de sistemas complexos.

Como trabalhos futuros desta proposta, pode-se destacar: i) construção de uma ferramentas que auxiliem a construção do sistema; ii) proposição de outros modelos, tais como: modelos baseados em outras metodologias, modelos baseados em frameworks para construção de sistemas multiagentes etc.; iii) definição de uma metodologia para a construção de sistemas multiagentes baseado na utilização desses modelos.

\section{Referências}

[1] M. Wooldridge, An Introduction to MultiAgent Systems - Second Edition, John Wiley \& Sons, 2009.

[2] N.R. Jennings, "On agent-based software engineering," Artificial Intelligence, vol. 117, Mar. 2000, pp. 277-296.

[3] M. Wooldridge and N.R. Jennings, "Intelligent Agents: Theory and Practice," Knowledge Engineering Review, vol. 10, 1995, pp. 115-152.

[4] M. Wooldridge and P. Ciancarini, "AgentOriented Software Engineering : The State of the Art," Agent-Oriented Software Engineering, vol. AOSE 2000, 2001, pp. 1-28.

[5] F. Zambonelli and A. Omicini, "Challenges and Research Directions in Agent-Oriented Software Engineering," Autonomous Agents and MultiAgent Systems, vol. 9, Nov. 2004, pp. 253-283.

[6] C. Bernon, M. Cossentino, and J. Pavón, "Agentoriented software engineering," The Knowledge Engineering Review, vol. 20, Jan. 2006, p. 99.

[7] F. Zambonelli, N.R. Jennings, and M. Wooldridge, "Developing multiagent systems: The Gaia methodology," ACM Transactions on Software Engineering and Methodology (TOSEM), vol. 12, 2003, p. 317-370. 
[8] J.F. Hübner, O. Boissier, and J.S. Sichman, "Programming MAS reorganisation with MOISE," Dagstuhl Seminar on Foundations and Practice of Programming MultiAgent Systems, J. Meyer, M. Dastani, and R. Bordini, eds., 2006.

[9] P. Giorginix, M. Kolp, J. Mylopoulos, and M. Pistore, "The Tropos Methodology: An Overview," Information Systems Research, 2004.

[10] E. de Barros Costa, "Um modelo de ambiente interativo de aprendizagem baseado numa arquitetura multi-agentes," 1997.

[11] L. Cabac and D. Moldt, "Formal semantics for AUML agent interaction protocol diagrams," Agent-Oriented Software Engineering V, 2005, p. 47-61.

[12] Q. Bai, M. Zhang, and K.T. Win, "A Colored Petri Net Based Approach for Multi-agent Interactions," Proc. of 2nd International Conference on Autonomous Robots and Agents, Palmerston North, New Zealand, Palmerston North: 2004, pp. 152-157.

[13] M. Nowostawski, M. Purvis, and S. Cranefield, "A layered approach for modelling agent conversations," 2001.

[14] Z. Yu and Y. Cai, "Object-oriented Petri nets based architecture description language for multiagent systems," IJCSNS, vol. 6, 2006, pp. 123 131.

[15] F. Bellifemine, G. Caire, and D. Greenwood, Developing Multi-Agent System with JADE, John Wiley \& Sons, 2007.

[16] T. Murata, "Petri nets: Properties, analysis and applications," Proceedings of the IEEE, 1989.

[17] J. Billington, High-level Petri Nets-Concepts, Definitions and Graphical Notation, 2002.

[18] K. Jensen, L.M. Kristensen, and L. Wells, "Coloured Petri Nets and CPN Tools for modelling and validation of concurrent systems," International Journal on Software Tools for Technology Transfer, vol. 9, Mar. 2007, pp. 213-254. 